International Journal of Engineering \& Technology, $7(1.1)(2018) 240-242$
International Journal of Engineering \& Technology
Website: www.sciencepubco.com/index.php/IJET
Research paper

\title{
Automated irrigation system using sensors and node micro controller unit
}

\author{
P. Shanmukha Sai Teja *, M. Vineel, G. Manisha, S. Satyanarayana \\ Student, Department of Computer Science and Engineering, Koneru Lakshmaiah Education Foundation \\ *Corresponding author E-mail: shanmukhasaiteja24@gmail.com
}

\begin{abstract}
Internet of things IOT is a network of systems connected within the systems, systems or devices like smart phones, sensors, computer. Internet of things connects all the surrounding devices to internet that is internet of things. Smart devices is an electronic device that can take logical and intelligent decisions on its own. Sensor are small chip that reacts to the sensed activities. Transfer of data is also done in IOT the data which is taken from sensors is sent through cloud database and again can be retreated for the necessary response. The medium of both smart device and database is internet. In this project main motto is to record the changes and information of agriculture field. This makes the easy way to cultivate the field in protective shield. Here we get the details of soil moisture, temperature, humidity, and periodic information of water supply to field. In agriculture water is the main source to a good healthy field. This project helps us to make use of water in efficiently in agriculture fields. Also farmer ids safe from many incoming dangers like thunder strike, snake bite and so on. Introducing technology to farmer to operate his field through his phone. This IOT project helps the farmer to collect the information about water level, soil moisture, humidity, soil fertility, and monitoring of crop allows to find weed, pest detection, animal entry into field, and growth of crop. Arduino is an open and free source hardware model used to dump software. It reads input signals of sensors and in response the output activation of things like motor, light. Sensors which senses the variation in different mediums of atmosphere inputs analogue to digital outputs. Think speak is open source cloud can store and retrieve data of sensors by local area network.
\end{abstract}

Keywords: Automation; Node Mcu Wifi; Irrigation; Sensors; Internet; Water Resource; Crop.

\section{Introduction}

IOT internet of things is platform where all smart devices are connected to internet to coordinate each other where there is no external help is required. [1]The internet of things is changing the world much with and then, the way we drive how we purchases sophisticated physical world sensors and chips are embedded in the physical thing that surrounded us, each of these will transfer and share data. This internet of things work in real time application like manufacturing machines, traffic analysis, and controlling our health this type of internet things platform that reverse the data and allow the simple language to smart devices and applications to communicate each other. This project is efficient to manage agricultural crop filed and low cost and reduces the working cost and save energy of working men. This project technology is based on wifi Arduino module to for wireless sensors. Water management through intelligent machines by use of soil moisture sensor by detecting moisture in soil it is designed for smart irrigation. This design is uses wifi data transfer to database. There is another de- sign which uses GMS Global System for Mobile Communications, the project contains both hardware and software model and network protocol with different tasks. It is compact in size and low weight with good performance and operation and improves productivity of field.

Existing method: There are many projects with same aim of application, but in different methods like operating through Arduino, micro processor with GPRS modem etc. the operation of this goes like this via message controlling or through pure automation. Innovation method: In our project we just made it simple and low cost equipment. We used mini Arduino node MCU with Wifi module and inbuilt humidity and temperature sensor, soil moisture sensor in addition to water level sensor to it. The whole project is automated itself. If the moisture in soil is low then the irrigation to crop field starts i.e the supply of water starts. All the data is transfer to cloud via wifi and represented in graphical analysis time to time. 
Graphical representation:

\section{Smart agriculture using IoT}

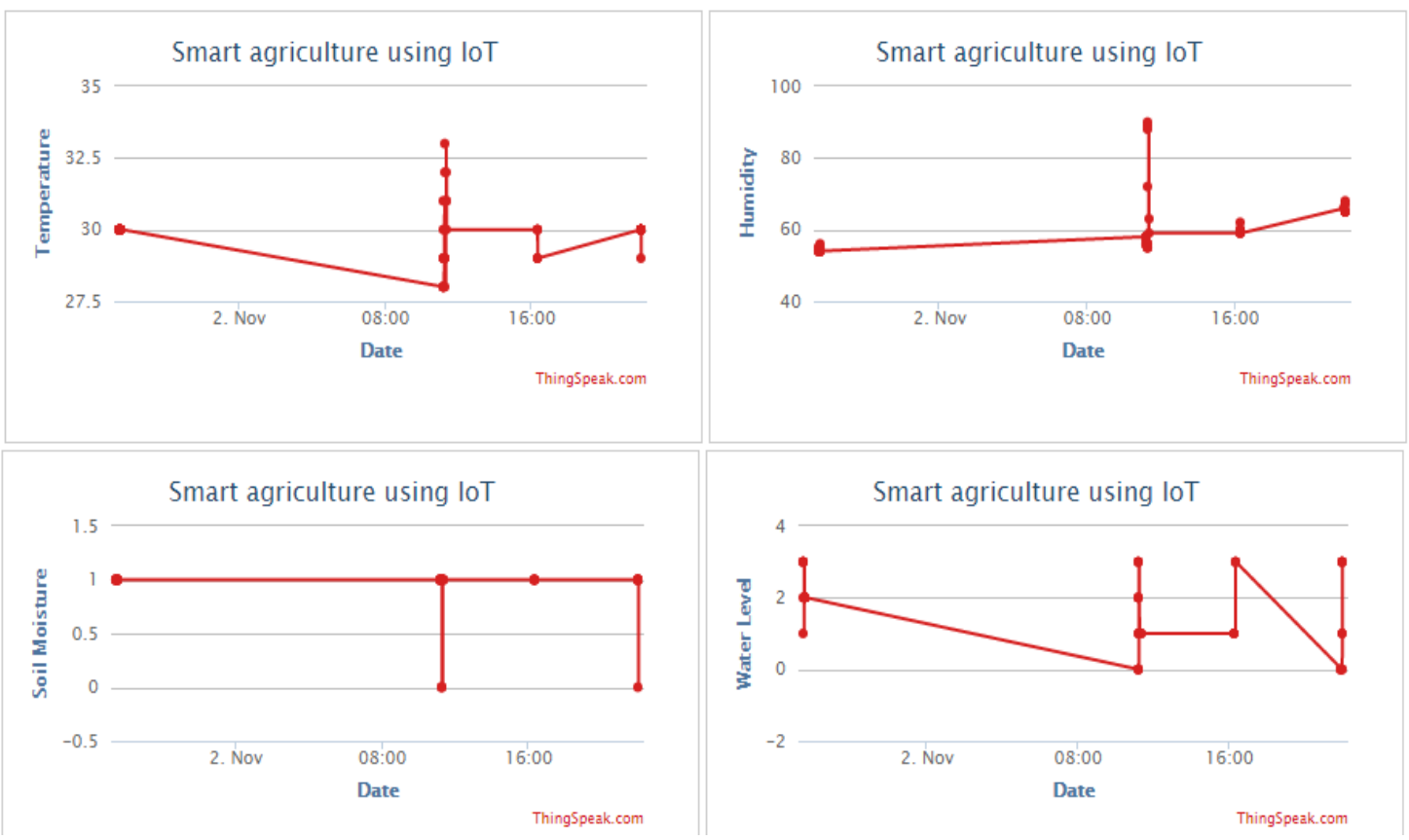

Fig. 1: Graph

\section{Applications}

Now-a-days agriculture is a risky action in hope of good result but weather, water scarcity, soil fertility; pesticides are the main problem for crop fields where they were spoiled. But internet of things is making agriculture beneficiary with few new applications of IOT. [3] Water management the adequate water supply is much for agriculture. Excess or shortage of water will kill crop. IOT makes better water management possible when couple with sensor, data and other machinery like automated water sprinkling, precision agriculture, soil quality, ect. Integrated Pest Management or Control, observation, Inspection, Identification, Record Tracking and automatic spraying of pesticides at right time. Remote Crop monitoring, Climate monitoring and Forecasting, Food production and safety with Smart Logistics and warehousing, Collection data and analysing every minute inch of crop and its growth and thus making it easy to predict the crop.

\section{Implementation}

Node wifi modem MCU is an open supply IOT platform.[5] It uses the AMBEDED C language. The project is the basic of Ardiuno board, and engineered on the ESP8266. nodeMCU uses several open supply comes. The Node wifi MCU runs on the ESP8266 Wi-Fi, and hardware that supported the ESP8266 module.54 milli meter pitch headers. The Node wifi MCU board uses the $\mathrm{CH} 340 \mathrm{G}$ USB/UART device chip. you may ought to transfer and install the correct driver to induce going with the event. An easy thanks to communicate with the LUA interpreter on the ESP8266. It permits you to perform straightforward tasks. as an example, set the SSID and watchword for your wireless router therefore it can hook up with your network via graphical user interface. you'll conjointly browse out or set the standing of its GPIO ports. Get information just like the information processing address or the chip ID, or transfer files.

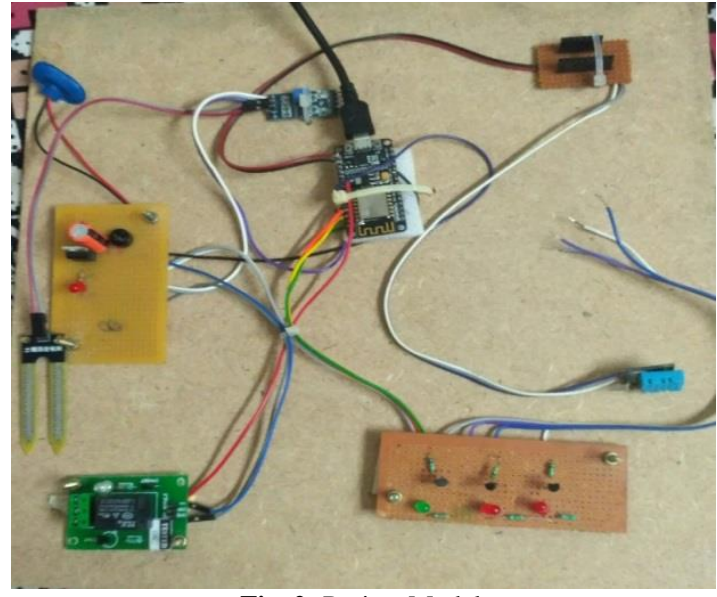

Fig. 2: Project Model

[9]Yet, you'll moreover try Explorer which features a additional up to date computer program. It conjointly has syntax highlight on LUA code. You can notice some usage examples here to begin your development activities [3] BC547 semiconductor One part that has revolutionized the technology at intervals the past decade is known by its name semiconductor unit. Transistors have enabled variety of humankind's biggest leaps in technology. Transistors actually helped begin age and it conjointly revolutionized computer style and spawned a whole business in California's aptly-named geographic area. Let's see what a semiconductor is all regarding It is named as semiconductor that is of 2 terms: "transfer-of-resistor." It implies that the inner resistance of semiconductor transfers from one worth to a different values counting on the biasing voltage applied to the semiconductor. so it's called transfer resistor: i.e. TRANSISTOR. 


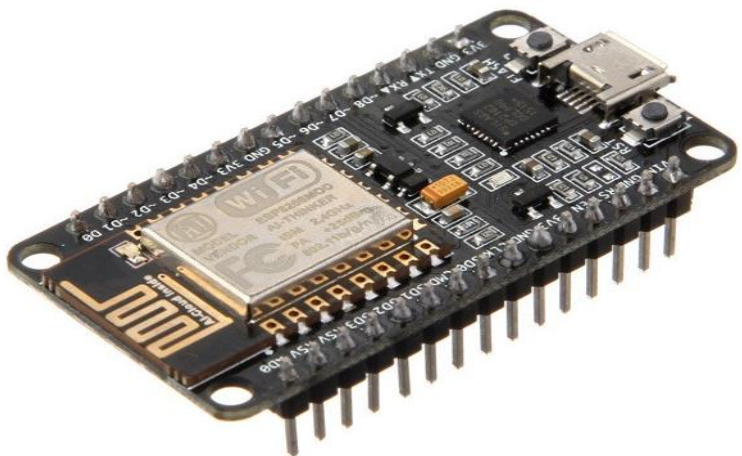

Fig. 3: Wifi Node Mirco Controller Unit.

A semiconductor may be a semiconductor unit accustomed amplify and switch electronic signals and wattage. Transistors works splendidly for a pc production. [8] With good engineering, transistors facilitate computers power though vast number of calculations in an exceedingly short time. The easy switch operation of transistors is what permits our pc to complete massively complicated tasks. In an exceedingly pc chip, transistors switch between 2 binary states zero and one this can be the language of computers. [10] One pc chip will have legion transistors along with regular switch. Semiconductor is made from element found in sand which is called silicon which is not a good conductor for electricity. Electrons have a charge, silicon is treated with n-type and conjointly referred to as NPN semiconductor.

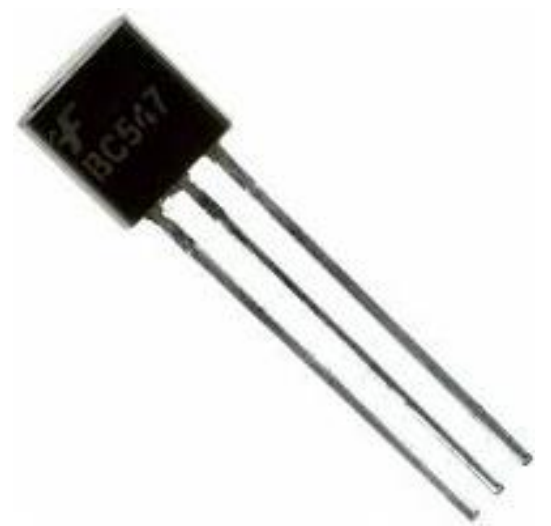

Fig. 4: BC547Semiconductor.

We will conjointly dope $\mathrm{Si}$ with alternative impurities like atomic number 5, gallium, and metallic element. Si treated this fashion can lose some electrons, therefore electrons in near materials can tend to flow into it.[2] an absence of electrons is that the same issue as a charge, therefore we tend to decision this type of Siptype (positive type) and conjointly referred to as PNP semiconductor. Brief Introduction to NPN semiconductor The NPN semiconductor are often employed in 2 totally. Together with alternative electronic parts, like resistors, coils, and capacitors, it are often used because the active part for switches and amplifiers. Like all alternative NPN transistors, this sort has an emitter terminal, a base or management terminal, and a collector terminal. In an exceedingly typical configuration, the present flowing from the bottom to the electrode controls the collector current. a brief vertical line, that is that the base, will indicate schematic for an NPN transistor, and therefore the electrode, that may be a diagonal line connecting to the bottom, is AN point inform removed from the bottom.

\section{Conclusion}

This paper describes about the low power smart irrigation system with wed based and application based with tracking temperature, humidity, water level, and water pump on and off timings are recorded. The hardware is implemented with a programmed node micro controller unit, soil moisture sensor, temperature and humidity inbuilt sensor, water level sensor, relay and a DC motor to pump water to field. By using this technique, any type of remote gadgets can be programmable micro controller to send and control data for handling supplies over internet.

\section{References}

[1] Sänket Salvi; S. A. Framed Jain; H. A. Sanjay; T. K. Harshita; M Farhana; Naveen Jain; M. V. Suhas "Cloud based data analysis and monitoring of smart multi-level irrigationsystem using IoT" 2017 International Conference on I-SMAC (IoT in Social, Mobile, Analytics and Cloud) (I-SMAC)

[2] P. Rajalakshmi; S. Devi Mahalakshmi "IOT based crop-field monitoring and irrigation automation" 2016 10th International Conference on Intelligent Systems and Control (ISCO) Year: 2016 https://doi.org/10.1109/ISCO.2016.7726900.

[3] O. M. Grant, M. J. Davies, H. Longbottom, and C. J. Atkinson,Irrigation scheduling and irrigation systems: Optimising irrigation efficiency for container ornamental shrubs," Irrigation Sci., vol. 27, no. 2, pp. 139-153, Jan. 2009. https://doi.org/10.1007/s00271 008-0128-X.

[4] Y. Kim, J. D. Jabro, and R. G. Evans, "Wireless lysimeters for realtime online soil water monitoring," Irrigation Sci., vol. 29, no. 5, pp. 423-430, Sep. 2011. https://doi.org/10.1007/s00271-010-0249-

[5] http://www.handsontec.com/pdf_learn/esp8266-V10.pdf, ESP8266 Lua Nodemcu WIFI Module. Handsontec.

[6] https://cdnshop.adafruit.com/productfiles/2471/0AESP8266_Datas heet_EN_v4.3.pdf.adafrui.

[7] DHT11 Humidity \& Temperature Sensor D-Robotics UK (www.droboticsonline.com)

[8] gutiérrez et al.: automated irrigation system using a wsn and gprs module. ieee transactions on instrumentation and measurement, vol. 63, no. 1, january 2014

[9] Y. Kim, R. Evans and W. Iversen, "Remote Sensing and Control of an Irrigation System Using a Distributed Wireless Sensor Network", IEEE Transactions on Instrumentation and Measurement, pp. 13791387, 2008.

[10] Agricultural crop monitoring using IOT - a study, 2017 11th International Conference on Intelligent Systems and Control (ISCO), Dr. D.K. Sreekantha, Kavya.A.M Professor, Department of Computer Science and Engineering, NMAM Institute of Technology, Nitte, Karnataka, India, sreekantha@ nitte.edu.in. 